\title{
Disseminating early years research: an illustrative case study
}

\author{
Siobhan O'Connor, Sinead McGilloway, Grainne Hickey and Melanie Barwick
}

\begin{abstract}
Purpose - This paper aims to outline a knowledge translation (KT) case study undertaken as part of a multi-component research programme aimed at evaluating new parenting supports in the earliest years. The study aimed to: explore the influencing factors relating to research use in an early years context; and to use the findings, at least in part, to execute an integrated KT plan - to promote stakeholder engagement, greater research visibility and to enhance the understanding of findings emerging from the research programme.

Design/methodology/approach - A mixed-methods study was embedded within a large-scale, longitudinal research programme. In the present study, a national survey ( $\mathrm{n}=162$ ) was administered to stakeholders working with children and families throughout Ireland. A series of oneto-one interviews were also undertaken $(\mathrm{n}=37)$ to amplify the survey findings. Also, one focus group was carried out with parents $(\mathrm{n}=8)$ and one with members of the research team $(\mathrm{n}=3)$. Several dissemination strategies were concurrently developed, executed and evaluated, based partly on survey and interview findings and guided by the knowledge translation planning template (Barwick, 2008; 2013; 2019).

Findings - The main factors influencing the dissemination of evidence, as identified by the stakeholders were: a lack of resources; an under-developed understanding of research use and dissemination; insufficient collaboration and communication; and conflicting stakeholder priorities. Despite these challenges, the research programme was found to benefit from a multi-component KT plan to achieve the outlined dissemination goals.

Practical implications - The KT planning process allowed the research team to be more accountable, introspective and to work more efficiently. This helped increase the likelihood of more targeted and successful dissemination of the research findings, delivering a better return on research investment.

Originality/value - This is the first study of its kind (to our knowledge) to provide important insights for stakeholders in Ireland and elsewhere about how to improve the dissemination process. Effective KT planning can ultimately help to bridge the research-policy-practice gap and enable the effective translation of high-quality evidence in the early years' sector to enhance outcomes for families in the shorter and longer-term.
\end{abstract}

Keywords Prevention, Dissemination, Intervention, Translation, Parenting, KT

Paper type Case study

\section{Introduction}

There are significant investments and continuing global interest in evidence-based parenting programmes and their impact on outcomes for children and families (Hickey et al., 2018; Hutchings et al., 2017; Leijten et al., 2015). As a result, many governments have developed policies advocating for the use of evidence-based parenting programmes including, for example, Better Outcomes, Brighter Futures in Ireland, Every Child Matters in the UK and the National Framework for Protecting Australia's Children. Despite the allocation of considerable funding internationally to expand knowledge in evidence-based research, it is not always effectively shared or implemented as intended in child and family services (Powell et al., 2017).
Siobhan O'Connor, Sinead McGilloway and Grainne Hickey are all based at the Department of Psychology,

Maynooth University, Maynooth, Ireland. Melanie Barwick is based at The Hospital for Sick Children, Toronto, Canada.

Received 30 April 2020 Revised 21 November 2020 Accepted 25 November 2020

Authors acknowledge with thanks the funding and support provided for this study by the Health Research Board. Authors extend their warmest thanks to the participants in the surveys, interviews and focus groups who kindly agreed to take part in this study. 
The field of implementation science has produced a growing body of international literature on how research is disseminated to a wide range of stakeholders (including practitioners, policymakers and service users) and the barriers and facilitators to implementing research to bridge the "knowledge-to-action" gap (Milat et al., 2011). The process of disseminating and implementing research evidence is commonly known as "knowledge translation" (KT) (CIHR, 2004). KT efforts aim to maximise the outcomes from research by ensuring stakeholders "are aware of and use research evidence to inform their health and health-care decision-making" (Grimshaw et al., 2012, p. 2).

Dissemination interventions or strategies comprise a broad range of isolated or multifaceted processes and practices used to achieve particular dissemination goals and which, ideally, involve engaging with multiple stakeholders throughout a research study (Barwick, 2016; Oliver et al., 2014). There is considerable literature on the effectiveness of dissemination strategies that aim to enhance research use; however, to date, there is no gold standard approach for selecting the most appropriate strategies (Stevens et al., 2014; Yamada et al., 2015). Varying dissemination goals, stakeholders and contexts can all require different strategies - for example, conference presentations can effectively share knowledge within the academic community but interactive workshops help to support behaviour change amongst practitioners (Edwards et al., 2019; Grimshaw et al., 2012). There is consensus that a multi-method approach (i.e. targeting several stakeholders using a variety of strategies) is thought to be associated with more successful dissemination as the more sources from which evidence emanates, the more likely it is to be heard, seen and acted upon (Kernohan et al., 2018; Li et al., 2018).

Public health researchers recognise the importance of engaging with stakeholders and communicating their research evidence beyond academic publications targeted at researchers. However, most admit to using opportunistic and haphazard dissemination practices in the later stages of the research, usually based on strategies used in the past and often neglecting non-academic stakeholders (Kernohan et al., 2018). Although some researchers engage in few researchers engage in KT planning to tailor, track and evaluate the impact of related strategies (Lombardi, 2018; Ngamo et al., 2016). Prospective and considered KT planning is likely to ensure more structured and effective research exposure and engagement which can ultimately increase research utilisation and impact (Barwick, 2016; Cambon et al., 2017).

In terms of research impact, there is a growing emphasis on assessing the effects of evidence on policy, practice and society, aside from knowledge uptake and implementation (Geddes et al., 2018). For instance, many health research funders (e.g. the Health Research Board [HRB] Knowledge Exchange and Dissemination Scheme in Ireland and the CIHR Planning and Dissemination grants in Canada) are now encouraging and even requiring investigators to demonstrate how they can share their findings in practical and usable ways (Barwick, 2016). The increased popularity of assessment schemes such as the Research Excellence Framework or the Knowledge Exchange Framework in the UK evaluate impact in terms of the reach (the measure of accessibility) of research with intended stakeholders, as well as the significance or usefulness of the findings (Kings College London and Digital Science, 2015). Thus, the influence of research findings can be assessed, at least in one way, by recording the number of individuals/organisations who accessed, understood or interacted with a piece of evidence (insofar as this can be evaluated) (Hill and McAlpine, 2019).

Ultimately, health research evidence cannot have a positive impact unless it is effectively communicated to the intended stakeholders. The dissemination process can be maximised through executing a context-specific KT plan early in a research project (Barwick, 2016; Cambon et al., 2017). 


\section{Aims and objectives}

The overarching aim of this research was to undertake a detailed case study that involved developing and executing a KT plan embedded within the context of a five-year research programme undertaken to assess the implementation and effectiveness of two wraparoundinspired service models designed to promote child and family well-being in the early years (Hickey et al., 2020; Leckey et al., 2019). For example, one of these models involves the delivery of a number of service elements (e.g. baby massage and paediatric first aid) to 106 parent and baby dyads during the first two years of life. The research was conducted in collaboration with multidisciplinary service providers such as public health nurses, family support workers and social workers.

The specific objectives of the current study were to: identify and outline the factors that influence dissemination effectiveness according to the perspectives, preferences and needs of a range of stakeholders from an early years context in Ireland; use some of the findings from the above to inform the KT plan for the research programme and to evaluate, insofar as possible, the selected dissemination strategies using key impact indicators.

\section{Method}

This mixed-method KT case study comprised a number of separate but related activities including a stakeholder analysis; an online survey of key stakeholders; a series of interviews and focus groups with key informants; the design and execution of several dissemination strategies informed by stakeholder input; and the ongoing evaluation of dissemination strategies to provide an understanding of evidence use and to determine whether stakeholders benefitted and how.

\section{Participants and settings}

Stakeholders were recruited using a mix of purposive and snowball sampling and were identified from the following stakeholder groups within the child and family sector in the Republic of Ireland (and Northern Ireland): policymakers; practitioners/service managers; researchers/Principal Investigators and parents.

\section{Procedures}

A stakeholder analysis was carried out to identify stakeholders to participate in the survey, key informant interviews and focus groups (Figure 1). The stakeholder analysis involved the development of a quadrant matrix that prioritised and mapped a list of stakeholders identified by the research team. These stakeholders were then grouped based on their relative influence and/or interest in the area of research use in the child and family sector (Eden and Ackermann, 1998). Most efforts were focused on stakeholders mapped in the high interest, high power group.

Following the stakeholder analysis, the researcher generated a list of stakeholder email addresses via Google searches (prior to the enforcement of the General Data Protection Regulations, 2018). Prospective stakeholders were invited via email to take part in the study; this included a link to an information sheet, consent form and the online survey. Fully informed written consent was sought from all stakeholders and they were also assured that any identifiable information would be removed. Stakeholders were also invited to circulate the survey to their colleagues or other relevant stakeholders whom they considered might be relevant. A total of 433 stakeholders were contacted, 37\% $(n=162)$ of whom responded. Following a request at the end of the online survey, seven stakeholders volunteered to take part in a one-to-one interview with the researcher. In total, 80 other prospective interview participants who were identified from the initial stakeholder analysis were invited via email to take part in key informant interviews based on their ability (and willingness) to provide more 


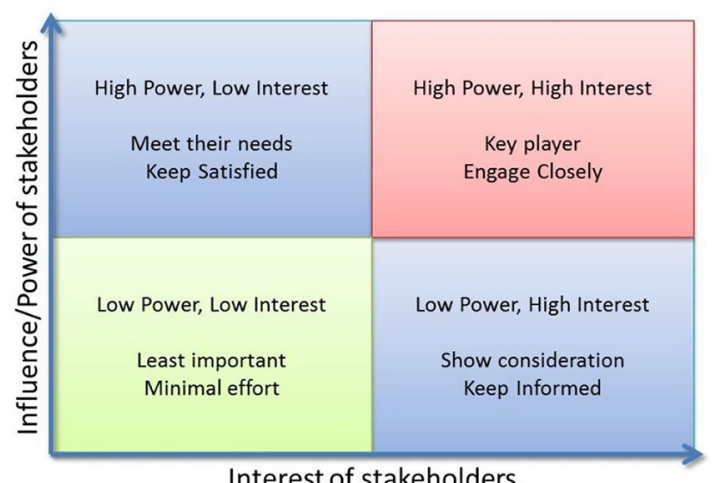

Source: Eden and Ackermann (1998)

in-depth input on experiences of research utilisation (e.g. senior managers/policymakers/ principal investigators). In total, $41 \%$ of these stakeholders agreed to take part in the research.

\section{Quantitative data collection measures}

Research utilisation survey. A 68-item questionnaire-based survey was - administered using Qualtrics Survey Software. The survey included a mix of five-point Likert scale questions (e.g. 1, "strongly agree"; 5, "strongly disagree"), multiple-choice answers and open-ended questions. This cross-sectional survey was designed to collect information on: demographic information; definitions of evidence; attitudes towards research; organisational culture of research use; experience with accessing, applying and disseminating evidence; and thoughts/views about factors that shape evidence use.

Survey items were adapted from existing measures used to investigate barriers and facilitators to research utilisation (Canadian Foundation for Healthcare Improvement, 2014; Landry et al., 2001). Qualtrics algorithms were configured so those specific survey items were displayed only to those identified as working in practice, policy or "other" (e.g. "Which of the following have you used to access evidence"?) and certain survey items were presented to those identified as working in research/academia (e.g. "Which of the following have you used to disseminate your research"?). The survey took approximately $20 \mathrm{~min}$ to complete. Non-respondents were sent two reminder emails in the month following the initial email to optimise the response rate.

Dissemination strategy evaluation measures. Several brief feedback forms were developed and distributed following knowledge-sharing and training events. These pen-and-paper measures included five-point Likert-scale structured questions and open-ended questions relating to the perceived usefulness of the strategy; stakeholders' knowledge status pre- and post the strategy; satisfaction with the strategy; and any intent to use or adapt the knowledge following the event. These measures were completed anonymously, took approximately 5 min to complete and were collected and collated once the strategy was complete.

\section{Interview/focus group schedules}

Key informant interviews/focus groups. Semi-structured interview and focus group protocols were tailored for each stakeholder group to supplement and amplify the survey 
findings and to explore individual experiences and contextual factors that inhibit and encourage evidence use. Interview questions were based on previous literature and the survey findings. Both focus groups and interviews ranged in length from approximately 20 to $45 \mathrm{~min}$ and were carried out by the same researcher, audio-recorded (with consent) and transcribed verbatim.

Parent advisory panel. A parent advisory panel of eight parents who took part in the research programme was convened to gather feedback on three dissemination strategies (i.e. an academic paper, research summary booklet and the webpage), to assess their usefulness for parents/families and to learn how best to communicate research to these stakeholders. All parents were provided with a tailored information sheet and completed a consent form prior to participating in the advisory panel.

\section{Dissemination strategy indicators and evaluation}

The knowledge translation planning template (KTPT) (Barwick,2008, 2013, 2019, www. sickkids.ca/pdfs/Learning/58366-58366-KT_Template.pdf) was used to guide the KT planning and evaluation. The KTPT is an evidence-informed planning tool for research dissemination that guides users through 13 core components of KT planning (Figure 2) including identification of main messages; targeted stakeholders; dissemination goals; dissemination strategies and indicators of dissemination impact and evaluation metrics.

The dissemination strategies used in this study were selected to align with the dissemination goals and preferences of the stakeholder groups, informed by the KTPT (Barwick,2008, 2013, 2019); and evidence on their effectiveness (Oliver et al., 2014; Powell et al., 2017). The evaluation of the dissemination strategies was ongoing throughout the research programme, using standard indicators such as reach (e.g. the number of publications), usefulness (e.g. user satisfaction) and use (e.g. intent to apply) (Barwick, 2016). Online tracking analytics, i.e. Google Analytics, Mailchimp reports, Facebook Insights and Twitter Activity, were used to measure data (such as views, downloads and social media mentions) from the project webpage, e-newsletters and social media accounts. Evaluation metrics were analysed using Microsoft Excel and described using means and frequencies. Details on presentations, publications, e-mailing lists and sign-in sheets were logged (where possible) by the research team.

\section{Figure 2 Core components of the KTPT}

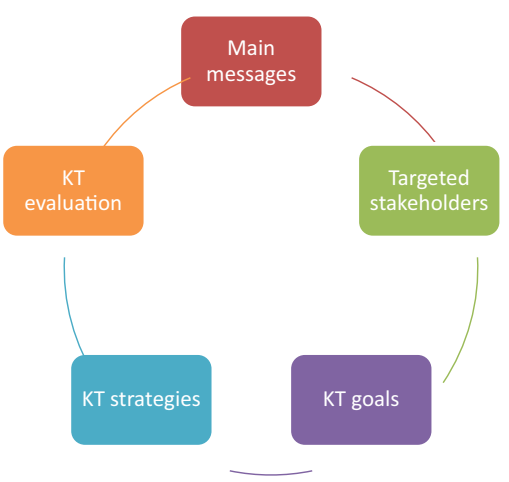

Source: Barwick $(2008,2013,2019)$ 


\section{Data analysis}

Qualitative and qualitative data were collected concurrently, analysed separately initially and then synthesised and integrated. The triangulation of findings helped to ensure overall rigour in the analysis process.

Survey responses were automatically collected in a secure database on the Qualtrics server and exported to SPSS (Version 25.0) at the close of the survey. Data were cleaned and analysed using appropriate descriptive statistics.

Qualitative data (including data generated through open-ended questions from the research utilisation survey and key informant interviews/focus groups) were stored and analysed using MAXQDA software (Version 12.0). A standard thematic analysis was conducted using a deductive approach - informed by Braun and Clarke (2006) established a priori in line with the research aims, previous literature and survey findings. An ongoing process of reading line-by-line and reviewing transcripts was carried out to identify common and divergent views, summarised with illustrative quotes organised around the theme of influential factors. Codes and emergent themes from the data were refined as necessary. Data saturation was considered to have been achieved at the point when no new themes were identified from additional interviews that would alter the interpretation of the results.

All of the data were coded and analysed by the primary researcher with ongoing consultation about the rationale of the theme with three team members experienced in qualitative research. Respondent validation was used by informing the interviewees of the primary factors identified from the survey results. Reflexivity was facilitated by debriefing the stakeholders following the interviews/focus groups and the researcher also repeated statements back to interviewees during the interviews to clarify understanding.

\section{Results}

\section{Participant characteristics}

The national survey sample consisted of 162 stakeholders working in research/academia ( $n=57)$, in practice - as a practitioner/service provider/manager $(n=67)$, in policy $(n=9)$ or in "other" fields such as community development and intermediary organisations $(n=29)$ (Figure 3). For purposes of analysis, the small number of policy stakeholders was combined with those identified as working in "other" areas $(n=38)$. A total of 37 one-to-one interviews were subsequently carried out with researchers $(n=7)$, practitioners $(n=7)$, policymakers ( $n=12)$, "others" (e.g. funders, intermediary organisations) $(n=3)$ and parents - as part of

Figure 3 Breakdown of the stakeholder groups that took part in the surveys/interviews/ focus groups

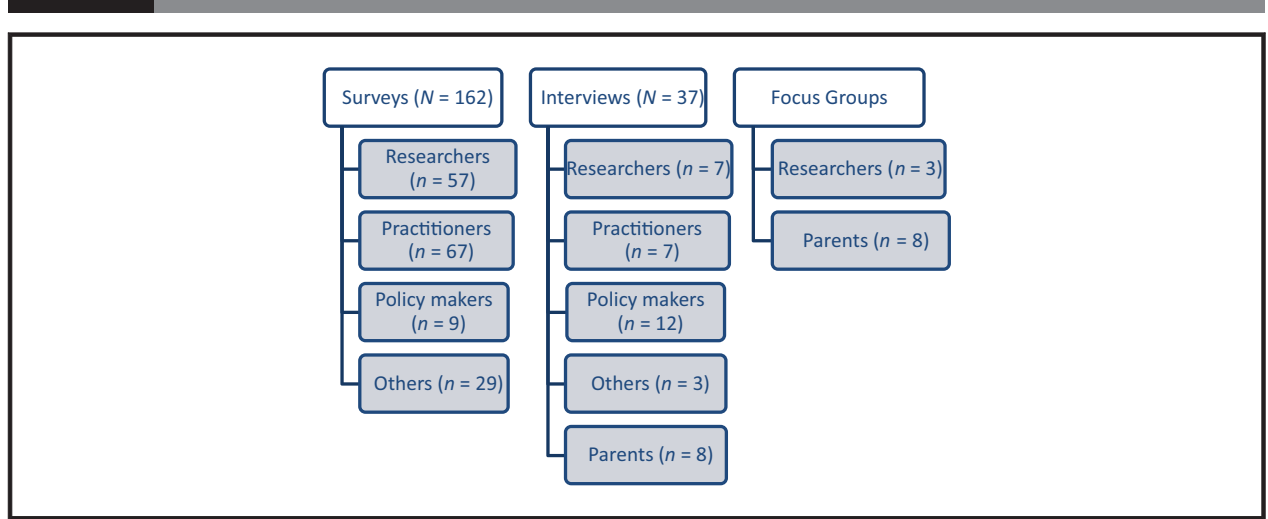


a Parent Advisory Panel $(n=8)$. Policymakers were more likely to respond to a request for an interview than a request to complete a survey and therefore, were targeted chiefly using this approach. Additionally, two focus groups were carried out, respectively, with a group of parents who did not take part in the research programme $(n=8)$ and with some members of the research team $(n=3)$.

Reassuringly, there was a high degree of overlap between the findings from both the survey and the qualitative interviews/focus groups. Thus, these are summarised in Figure 4. More detailed findings will be reported elsewhere. The dissemination strategies and their evaluation are described thereafter.

Key factors influencing research use in an early years context. Many contextual elements can influence how research evidence is shared and used amongst the intended stakeholders. The following factors were highlighted as the most influential in the early years setting and informed the development of the KT plan and the dissemination strategies.

Resources and accessibility. Overall, a lack of resources to facilitate access to and dissemination of research was identified by the majority of stakeholders, including researchers $(n=44)$, practitioners $(n=38)$ and policy/others $(n=27)$, as a primary factor influencing the use of research:

"Money is the issue and the control of money and all the other issues are subsequent to that"

(Practitioner [P] 2).

One key informant noted that "the biggest barrier is knowing [research evidence] is out there at all" (policymaker [PM] 11) while a large proportion of practitioners $(n=44)$ and policy/others $(n=21)$ identified accessibility to expensive academic journals as problematic. All stakeholders described not having the "luxury of time" in the working day to access, digest and disseminate research evidence in a timely and varied manner. Engaging in more dissemination would require more resourcing to allow staff the ability to access evidence through paywalls on academic journals, attend conferences and attend capacity-building training. Also, the research team acknowledged that: "having a dedicated person looking after KT has made a huge difference to this project" (Research Focus Group $[R F])$. This shows the value of investing in $\mathrm{KT}$.

Understanding and capacity-building. The capacity of stakeholders to interpret research findings and to engage in a variety of dissemination methods were also viewed as a barrier by practitioners $(n=37)$, policy/others $(n=29)$ and researchers $(n=22)$, respectively:

"[Practitioners] have no understanding of the power of evidence" (PM1).

The inclusion of technical jargon and statistics in journal articles and conference presentations was perceived to limit access to evidence for non-academic stakeholders. Enhancing stakeholder research appraisal skills through training supports was further

Figure 4 Key factors influencing research use within an early years context

Resources \& Accessibility

Understanding \& Capacity-building

Conflicting Priorities

Communication \& Collaboration 
identified as imperative for practitioners $(n=36)$ and policy/others alike $(n=21)$. Importantly, it was felt that there is a lack of support and training available in academic institutions to build researcher capacity to engage in dissemination. Therefore, any nonacademic dissemination is, perhaps seen as less important within academia and, yet, it is crucial for engagement and impact and should be fostered:

"We don't have the university's blessing to really pursue all those other kinds of knowledge translation activities which we should be doing" (RF).

Likewise, the research team acknowledged that engaging in effective and meaningful dissemination requires a different skillset from what they are trained in. Academic writing contradicts much of the KT literature that encourages dissemination through key point summaries, but "academic writing by its nature is not concise" and "woe betide you if you leave a detail out in an academic publication" (RF).

For the same reason, two key interviewees recommended KT training be incorporated into postgraduate programmes to educate researchers on how to disseminate findings in diverse ways "so researchers coming out of that process have those skills starting off" (Researcher [R] 21) and to strengthen their capacity for dissemination over the longer term.

Conflicting priorities. Respondents reported that roles, responsibilities and conflicting pressures could also influence how research is used in the early years' sector. Practitioners described researchers and policymakers as being "at a distance" from frontline issues and indicated that decision-making around early parenting supports was more likely to be dictated by short-term political demands than evidence-based research. Researchers, on the other hand, were perceived to be more focused on securing funding and producing publications for career development, which is "distinct from what is actually needed in the field" according to one research respondent (R6):

"[Researchers] care whether the research is cited again in another journal and lets just all keep each other in a job, citing each other's work, but does the research work actually care whether practitioners read their research" (R6).

Indeed, the academic infrastructure was considered to be an important barrier to dissemination. It can be difficult for researchers to strike a balance between producing time-consuming traditional research papers and engaging in more varied dissemination within the allocated time for a research project (i.e. before funding runs out). The research team acknowledged that researchers need to be very committed to complete the dissemination process, which often tends to go considerably beyond the end date of the actual project. Thus, it was seen as imperative that funders and academic institutions shift to measuring scholarship by recognising dissemination and impact (particularly the value of relationship-building) in addition to the more traditional publications and citations:

"You spend an inordinate amount of time planning and designing and speaking to collaborators and you have nothing to show for it. If engagement is done well, we should be able to, theoretically anyway, do more effective knowledge translation" (RF).

However, a huge push in Ireland and elsewhere towards engaged research was also recognised. Grant schemes (such as the aforementioned HRB Knowledge Exchange and Dissemination Scheme) that build in resources to encourage and incentivise proactive dissemination, were commended as having helped to "change the whole interface of research" (R24). These kinds of incentives were viewed as important for further development in $\mathrm{KT}$ and embedding it throughout a research project.

Communication and collaboration. A reliance on one-way linear communication was also considered to be a substantial barrier to dissemination by all stakeholder groups. One key informant stressed that researchers need to be more transparent and engage with the stakeholders that contribute to their research findings: 
"For God's sake researchers - get it together and feedback and show people how important they are and what changes their involvement has created for the better rather than saying thanks for your input" (R24).

Therefore, a key strength of this study was the inclusion of parents in the evaluation process to help determine how best to communicate research findings to these stakeholders. One parent from the Parent Advisory Panel noted:

"It was kind of nice to see just the results because I really felt like I benefitted from doing the group" (PT [Parent] 5).

The survey found practitioners and policy/others working in early years setting most commonly accessed research findings through research papers $(n=55 / n=29)$ and grey literature (such as reports, summaries and newsletters) $(n=54 / n=31)$. Conferences and workshops were also commonly used by both practitioners and policymakers alike as a means of sourcing evidence. Although stakeholders regularly access evidence through traditional papers, there appears to be a yearning to move away from this type of text-heavy and formal dissemination:

"Nobody is going to read documents that are 50 pages long. Those days are gone" (RF).

The use of varied and creative means of communicating evidence (e.g. summaries, infographics or online resources) - presented in an accessible, easy-to-read and "digestible" vital facilitator at every stage in the dissemination process. One key informant policymaker noted:

"What attracts me to a piece of work is that the summary of the document clearly outlines what the evidence is about, what kind of programme it is referring to, how it links to a policy area that I am working on" (PM15).

Parents, on the other hand, expressed a strong preference for face-to-face communication with a trusted party (e.g. another parent or a nurse) when accessing knowledge on parenting practices. The use of social media was also highlighted as a useful way to draw attention to research findings as the "phone is at your fingertips" (Parent Focus Group [PF]). Websites deemed trustworthy (such as the NHS or HSE) were also used by parents to access information on child and family health and well-being. However, perhaps, unsurprisingly, most parents in the focus group were not keen on academic publications for accessing information:

"You might only want a tiny piece of information out of that and you have to scan through pages and pages and pages just to get to the outcome or the conclusion" (PF).

In terms of collaboration, a sizeable proportion of researchers $(n=56)$, practitioners $(n=53)$ and policy/others $(n=35)$ surveyed or interviewed identified insufficient collaboration and a lack of cross-sector interaction as a major barrier to the sharing and utilisation of research evidence. All stakeholder groups alluded to the need for relevant parties to engage in more meaningful engagement to promote and facilitate effective dissemination through, for example, round table discussions as it is "hard to beat personal interaction" (p.m.11). Interestingly, despite claims by researcher participants $(n=36)$ of feeling disconnected from policymakers, the latter indicated their desire to be more included in research dissemination "maybe you are reaching out and we are not hearing" (p.m.11). Therefore, it is important for researchers to continually prioritise engaging and communicating with policymakers throughout the research process, by sending summaries and reports to the relevant departments and inviting representatives along to knowledge-sharing events, as advised by several key informant policymakers.

On a more positive note and despite an identified need for further collaborative efforts, it was indicated by several researchers and one practitioner that there are positive efforts 
within early years networks in Ireland to support the development and engagement of stakeholders - "[practitioners]meet once a month and it is kind of a peer learning group" (P28). It should be noted that, according to the research team, the current early years' focus can create competition when trying to communicate research findings to policymakers within "a very crowded field" (RF). Nevertheless, the importance of communicating findings and engaging with all target stakeholders cannot be underestimated.

Dissemination strategies - execution and impact. The influential factors identified from the quantitative and qualitative data were used, in part, to inform the development and execution of a series of dissemination strategies by advising the research team on the type of strategies to target particular stakeholders.

Each strategy is described in relation to core components of the KTPT (Barwick,2008, 2013, 2019) - the stakeholders, the message, the dissemination goal, the strategy and the impact indicator (reach and usefulness) (Table 1). The primary goals of each dissemination strategy were to promote stakeholder engagement and increase programme visibility. A secondary aim was to enhance the understanding of findings emerging from the research programme. Importantly, the execution of these strategies was constrained by the available budget and resources.

Knowledge-sharing events. Survey respondents and interviewees highlighted free interactive knowledge-sharing events as an effective means of communicating evidence and promoting informal discussions amongst stakeholders about the findings. The research team organised six knowledge-sharing events over five years. These were attended by a wide range of stakeholders $(M=23)$, primarily researchers $(M=7)$ and practitioners $(M=$ 20). All event attendees who provided feedback ( $n=43$ out of 105 ) reported the information was helpful and, on average, $96 \%$ reported the event increased their understanding of the research programme.

Conference presentations. Stakeholders stated they regularly accessed evidence by attending conferences. As expected, the research team also presented their findings at 23 national and international conferences in both poster and oral presentation formats. In terms of reach, these conferences targeted primarily researchers, although practitioners and policymakers were also represented. Overall, these events provided a useful opportunity for interaction and relationship-building with all stakeholders, albeit with a principal focus on researchers. Also, the Principal Investigator of the research programme was an invited speaker at several events, which further enhanced external interest in and awareness of, the research.

Table 1 Core components of the KTPT (Barwick, 2008, 2013, 2019) applied to the KT plan

\begin{tabular}{|c|c|c|}
\hline Strategy & Stakeholder group & Impact indicators \\
\hline Knowledge-sharing events & Practitioners researchers & $\begin{array}{l}\text { Reach (number of events and attendance) } \\
\text { Usefulness (satisfaction and intent to use) }\end{array}$ \\
\hline Conference presentations & Researchers & Reach (number of presentations and location) \\
\hline Publications & $\begin{array}{l}\text { Policymakers practitioners } \\
\text { researchers }\end{array}$ & $\begin{array}{l}\text { Reach (number of publications) } \\
\text { Usefulness (online engagement) }\end{array}$ \\
\hline Grey literature & $\begin{array}{l}\text { Policymakers practitioners } \\
\text { researchers } \\
\text { parents }\end{array}$ & $\begin{array}{l}\text { Reach (number of newsletters distributed/downloaded) } \\
\text { Usefulness (online engagement) }\end{array}$ \\
\hline Webpage and social media & $\begin{array}{l}\text { Policymakers practitioners } \\
\text { researchers } \\
\text { parents }\end{array}$ & $\begin{array}{l}\text { Reach (number of followers) } \\
\text { Usefulness (online engagement and social media coverage) }\end{array}$ \\
\hline KT skills training & Practitioners researchers & $\begin{array}{l}\text { Reach (attendance) } \\
\text { Usefulness and use (knowledge change generated and } \\
\text { intent to use) }\end{array}$ \\
\hline
\end{tabular}


Publications. Most of the stakeholders in this context still primarily access evidence through published peer-reviewed literature. At the time of writing, the research team had published their work in several different outlets $(n=7)$ (both traditional journals and non-academic publications) targeted to a range of stakeholders (additional papers are in submission/ preparation). Some of the publications were aimed specifically at stakeholders in the child and family sector in Ireland (e.g. Children's Research Digest as part of the Children's Research Network), while the remainder were aimed at an international readership (e.g. BMC Health Services Research which had an impact factor of 1.843 in 2017), so overall, there was varied targeted reach.

With regard to usefulness, Altmetric data were available for three of the publications and revealed they had a good attention score relative to other articles from the same journal published within the same time period, with two in the top 25\% compared to other research outputs scored. From the perspective of most of the Parent Advisory Panel, (again as expected) the sample academic paper was not as well-received as other formats because it was considered more time-consuming to read and difficult to understand:

"I had to read a couple of times to understand, you couldn't scan over it" and it was "full of research language, cold actually, clinical" (PT4).

Grey literature. The findings from the survey, interviews and focus groups indicated a need for research findings to be summarised in brief, user-friendly and visually-appealing formats. Therefore, the importance of grey literature is apparent when engaging with any stakeholder group. In response to this, the research team produced (to date) four project e-newsletters, four summary booklets and various reports to disseminate the research findings at various junctures throughout the programme. The e-newsletters were distributed to researchers $(M=$ 46.5) and practitioners $(M=50.3)$ primarily and also to policymakers $(M=11.25)$. Tracking data revealed the e-newsletters were opened by all stakeholder groups, with, on average, a $38.3 \%$ open rate, including two clicks on to the research webpage and five additional subscription requests to the newsletter. The tracking data increased with every issue, which indicated a growing engagement with and interest in, the research. Interestingly, the open rate was higher than the average according to the benchmark of 21.33\% (Mailchimp, 2019).

Summary booklets were distributed through the project webpage, e-newsletters, social media and at knowledge-sharing events. A sample booklet summarising the aims and findings of the research findings received an overwhelmingly positive response from all members of the Parent Advisory Panel, who found it to be a colourful and comprehensive yet concise account of the research:

"It was easy reading and you weren't puzzled either, it gave a good explanation of how the research panned out and the scale [...] I felt there was a lot of information in the small amount of reading" (PT5).

Project webpage. The research webpage is part of a research centre website that shares research findings as well as other news from the programme. Google Analytics reach indicators show how website traffic and engagement increased over time (Figure 5). Many users accessed the website through social media links, which shows the benefit of linking dissemination strategies to further promote the visibility of the research. Within the website, the research programme webpage was one of the most popular pages. The greatest number of weekly visitors to the website coincided with a highly publicised launch of the research programme's findings, which was featured in radio and newspaper media outlets at a national level.

In terms of benchmarks for webpage reach, it has been suggested that there is no ideal number of visitors; effective reach merely depends on whether the targeted stakeholders are engaging with the webpage content (Andrews, 2016). The number of visitors did increase over time, which suggests increased engagement. Also, the average time spent 


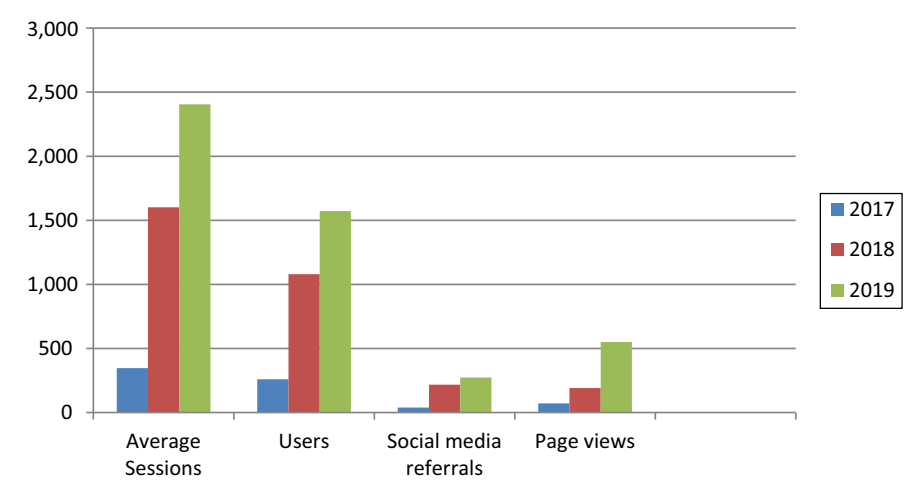

Note: *"Sessions" measure unique and individual visits to the website

on a webpage is an indication of the interest and value of the content to the user. The average time spent on this research webpage was $2.28 \mathrm{~min}$, which is marginally higher than the 2.11 min typically spent on higher education websites (Grzymkowski, 2019). All stakeholders stated the need for an online presence to share research findings and, more specifically, the Parent Advisory Group found the programme's webpage easy to navigate, visually appealing and useful as a "one-stop-shop for all reports" (PT2). However, the content was amended and abbreviated due to feedback suggesting it was a "bit textheavy" (PT3); and this may negatively impact the likelihood of parents accessing evidence through this forum.

Social media. The use of social media to share knowledge was also encouraged by stakeholders. A research Twitter account was created at the end of Year 2 (when the research had become more established) and a Facebook page was created in mid-Year 3 (based on the survey data) as a way to share research updates, relevant early years content and to interact with stakeholders nationally and internationally. In the final three years of the programme, Twitter followers nearly doubled from 370 to 602 . By comparison, a large-scale longitudinal research study in Ireland called "Growing Up in Ireland" -running for over 13 years - had approximately 1,000 followers at this time (Growing Up In Ireland, 2017).

Twitter analytics provide summary reports of activity and engagement (Figure 6). Impressions or the number of times a tweet appears in other users' timelines or search results, increased by 4,000 after the first year. Impressions are considered a low-level indicator of engagement, while "likes" (a user agrees with a post), "retweets" or "shares" (a user shares a post with their followers) and "comments" indicate higher engagement (Neiger et al., 2012). The "engagement rate" refers to the number of times a Twitter user interacts with a tweet and here, the rate was above $1 \%$ throughout the programme which is considered to be very good (Mee, 2019). This form of social media has proved to be a useful means of promoting the research programme and encouraging interaction with stakeholders.

By the end of Year 4, the research Facebook page had attracted only 23 followers and increased to 47 by 2019. In the final three years of the programme, Facebook tracking data revealed 3.5 page views, on average and an increase in the number of people reached (how often a post appeared on other users' timelines), from 20 to 150 . With only 46 engagements recorded from 2017-2019 characterised as posts with direct interactions including all clicks, comments, likes and shares, Facebook yielded a low level of reach and 


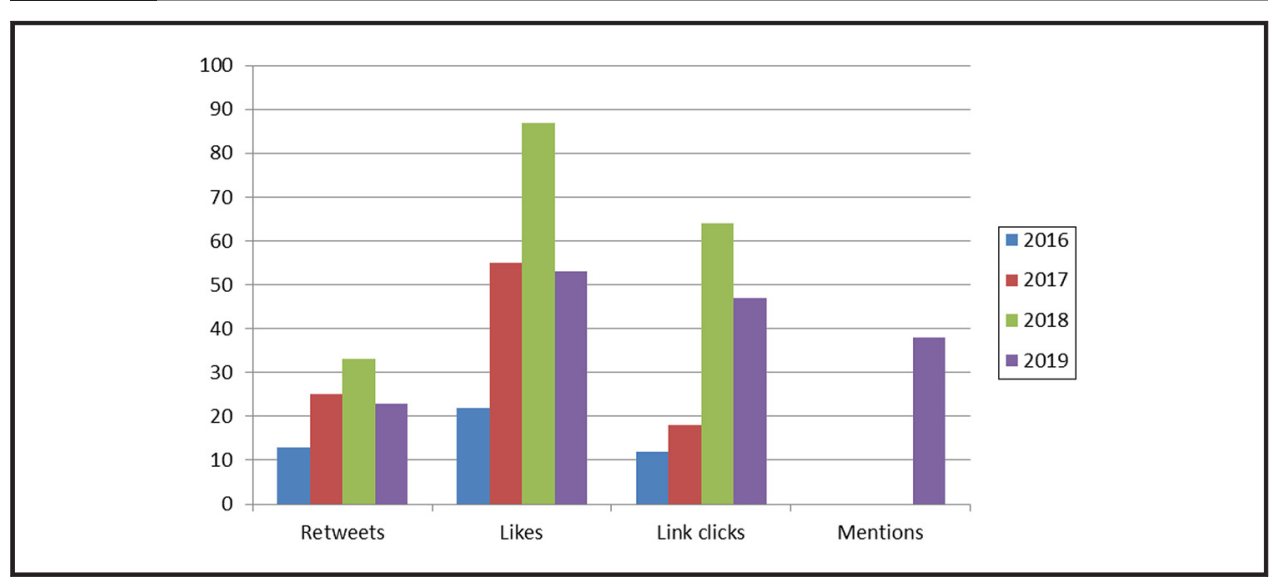

engagement for the research programme. Facebook and Twitter engagement differs in ways that may impact dissemination of research findings. For instance, Twitter enables users to engage through the use of hashtags (Zhang and Ahmed, 2019).

Knowledge translation skills training. Most of the stakeholders and members of the research team highlighted the need for KT training, which they reported as often a low priority in academia. The research team organised three KT skills training courses during the research programme, aimed at enhancing dissemination capacity, understanding and skills in how to disseminate findings using videos, plain language and how to engage in KT planning. KT training was advertised and promoted to the wider researcher and practitioner community through social media and email. The KT skills training was attended by a total of 40 stakeholders, primarily researchers. All of the attendees who completed feedback forms found the events useful and indicated they had improved understanding of KT and how to communicate research using accessible language and videos; 92\% of workshop participants reported improved dissemination skills following the training. Furthermore, $85 \%$ stated they would recommend the training to their colleagues and all intended to apply the knowledge and skills that they learned. The perceived value of the training was reflected in these terms and in their intent to develop a comprehensive KT plan using the KTPT (Barwick,2008, 2013, 2019); 73\% of participants reported they had never previously developed a KT plan for a research project.

The research team found the KT skills and capacity-building workshop informed the rest of the dissemination process:

"I wish we had done the KT training earlier" (RF).

Importantly, the team also acknowledged how their understanding of the dissemination process and the value of KT planning evolved throughout the research programme:

"I think that every single project should have a special KT piece and you should be thinking about it from the very beginning" (RF).

\section{Discussion}

The objectives of this study were to identify factors influencing dissemination effectiveness according to the perspectives and needs of a range of stakeholders in an early years context and to use these findings to inform a series of dissemination strategies as part of a KT plan for a research programme. 
The factors identified in this early years context mirror those identified in the wider health literature. For example, the availability of appropriate resources - particularly funding and time to access and deliver research - are considered paramount to achieving effective dissemination, particularly in health research (Margaryan et al., 2011; Tricco et al., 2016). The provision of free, accessible, jargon-free and varied options for communicating research (that are less time-intensive) were consistently recommended by the stakeholders in this study. The importance of promoting awareness and visibility of the research findings was emphasised. Indeed, research is perceived to be a waste of time, funding and resources for researchers if it gathers "digital dust" online (Green, 2019; Stevens et al., 2014). Also, capacity-building initiatives that - crucially - promote understanding amongst all stakeholders are deemed to be essential for improving research availability and accessibility (Cairney et al., 2016). The research team greatly benefitted from the KT skills capacity-building workshop but notably, this training was only made available through extra dissemination funding secured by the research team. These kinds of grants reflect a growing interest in and support for KT in Ireland and should be encouraged by all funding bodies to enhance research dissemination.

Threaded throughout the findings are a sense of disconnect and lack of meaningful engagement amongst stakeholders, particularly between researchers and policymakers, that has also been reported elsewhere in Europe, North America and Australia (Armstrong et al., 2013; Cambon et al., 2017; Oliver et al., 2014). Genuine and regular face-to-face interactions, as opposed to tokenistic interactions that simply satisfy funder requests, are vital to enhance research quality and increase the likelihood of research being understood (Kernohan et al., 2018). This kind of integrated KT approach - rooted in engagement and collaboration throughout the research process - can ultimately maximise research impact (Gagliardi et al., 2016). The research team felt the focus on KT helped them to be more proactive in collaborating and relationship-building with stakeholders and the KT plan helped the team become more aware of how to reach out to and engage with stakeholders through inviting them to knowledge-sharing events and by preparing user-friendly documents. However, the collective findings reported here suggest that all stakeholders must invest time, effort and commitment to achieve the long-term potential of collaborative relationships for future public health research.

Many of the barriers to research utilisation reported by stakeholders are related to the organisational contexts in which they work such as inadequate facilities, supports and administrative constraints (Elueze, 2015). The qualitative data suggested the academic world, in particular, does not always foster an environment that encourages effective dissemination. For instance, the research team highlighted the struggles of trying to balance varied dissemination strategies with other research tasks and duties alongside an ever-present pressure to produce traditional academic outputs. The wider political and social context also influences research use (Darker et al., 2018). In Ireland, a current strong focus on early intervention and prevention (including parenting supports) has, in turn, led to an increased interest in evidence-based programmes and related research. This is an important facilitator for promoting awareness of this research programme.

The use of the KTPT (Barwick,2008, 2013, 2019), in particular, was central to achieving optimal results and indeed, a number of authors have advocated for the use of a guide to orient a KT plan, particularly for the development and evaluation of dissemination strategies (Barwick, 2016; Cambon et al., 2017; Lombardi, 2018). The use of an evidence-based planning tool, in this instance, allowed the research team to be more accountable, introspective and transparent, to work more efficiently and, arguably increased the likelihood of more targeted and successful dissemination of the research findings. KT planning also allowed the research team to justify the resources spent on dissemination strategies, which can result in a more cost-effective programme. For example, the research summaries were favourably received by all stakeholders and were relatively low cost to 
disseminate, thereby indicating it does not require a huge financial investment to share evidence.

In terms of the early years' context, this case study contributes to and supports the growing international evidence base around the effectiveness of multi-faceted dissemination interventions (Yamada, 2015; Park et al., 2018). Web-based resources such as videos, websites, social media and e-newsletters, are increasingly used to build and strengthen awareness, reach and engagement with research evidence amongst widespread stakeholders (Van Eerd and Saunders, 2013) and they were also well regarded by the stakeholders in this study in both the quantitative and qualitative elements. There was a marked increase in research engagement and visibility over time, particularly from the more "non-academic" dissemination strategies such as the interactive knowledge-sharing events, grey literature, Twitter and KT skills training. The project Twitter account achieved good engagement indicators and reached a large number of followers when compared to the Facebook page, which informs future KT plans. The Facebook account did not appear to be a feasible part of the KT plan as the reach and engagement indicators were poor. This corresponds with Twitter being the most popular free platform for academic research that tends to be used more for professional purposes than Facebook (Zhang and Ahmed, 2019).

According to the survey responses, policymakers and practitioners access evidence most often through peer-reviewed publications. In contrast, the qualitative data revealed research summaries were preferred by all stakeholders. This suggests that, perhaps, traditional papers are more readily available for these stakeholder groups but they are not the preferred means of accessing evidence. Nonetheless, journal articles were still considered useful as part of a comprehensive dissemination intervention, satisfying current academic responsibilities. As long as traditional dissemination continues to be valued as strongly as it is in academia, researchers must continue to produce these outputs but could incorporate a proactive dissemination approach, e.g. including infographics on conference presentations or publishing journal articles in an open-access format.

The findings from this study also confirm the value, (but also the constraints), of measuring research impact in terms of indicators such as reach and usefulness. With increased pressure to demonstrate impact from funders, it would be useful for researchers to monitor research-related online data such as social media or altimetric data. However, it is not always feasible to evaluate or monitor every dissemination strategy (e.g. usefulness indicators from presentations) in terms of the dissemination goal. In the current study, data protection regulations prevented the collection of individual evaluative data such as details on each visitor to the webpage. Also, it is not always possible to track third party reach data (e.g. external websites sharing the research findings). However, overall these impact indicators were a useful way of assessing dissemination efforts.

There are limitations to this KT plan. Some of the dissemination strategies can be costly and/or labour-intensive to develop and maintain. Importantly, in terms of online dissemination, the level of stakeholder engagement and impact mirrored the amount of content and time that the research team invested in developing these dissemination strategies. Thus, these strategies, while broadly effective, require ongoing management and innovation which can present challenges for researchers in terms of leadership, dedicated time, financial resources and skills capacity, particularly when there is still an emphasis within academia on traditional forms of dissemination. Thus, any KT plan developed must be manageable within reasonable limits.

Although there are other dissemination case studies in the literature (Dew and Boydell, 2017; Home et al., 2015), this is the first study of its kind (to our knowledge) which provides a useful snapshot into the "how" aspect of the dissemination process, which could be transferable to future research programmes in Ireland and beyond. The findings suggest that research teams should use evidence-based KT planning tools (such as the KTPT; Barwick, 2008, 2013, 2019) 
to guide a comprehensive and feasible dissemination strategy that works best for their research project instead of approaching dissemination haphazardly. This case study illustrates the importance and value of KT planning, working together with stakeholders (including parents) throughout the research process to enhance dissemination (Fitzpatrick, 2012; Wathen and MacMillan, 2018). This is more likely to produce visible and impactful evidence, potentially deliver better returns on research investment and help researchers to leverage additional funding (Green, 2019). This "real world" example of the efforts of researchers to communicate their findings and promote their research early in the programme helped to build a broader understanding of the contextual infrastructure and the factors influencing evidence dissemination, albeit with some limitations. These kinds of approaches should ultimately help to more effectively bridge the research-policy-practice gap and enable the more effective translation of high-quality evidence in the early years' sector to enhance outcomes for children and families in the shorter and longer-term.

\section{References}

Andrews, A.L. (2016), "What is good traffic?", available at: https://amylynnandrews.com/what-is-goodtraffic/ (accessed 15 September 2019).

Armstrong, R., Waters, E., Dobbins, M., Anderson, L., Moore, L., Petticrew, M., Clark, R., Pettman, T.L., Burns, C., Moodie, M. and Conning, R. (2013), "Knowledge translation strategies to improve the use of evidence in public health decision making in local government: intervention design and implementation plan", Implement Sci, Vol. 8 No. 1, p. 121.

Barwick, M. (2008, 2013, 2019), "Knowledge translation planning template", available at: http:// melaniebarwick.com/knowledge-translation-tools/ (accessed 10 December 2019).

Barwick, M. (2016), "Building scientist capacity in knowledge translation: development of the knowledge translation planning template”, Technology Innovation Management Review, Vol. 6 No. 9, pp. 9-15.

Braun, V. and Clarke, V. (2006), "Using thematic analysis in psychology", Qualitative Research in Psychology, Vol. 3 No. 2, pp. 77-101.

Cairney, P., Oliver, K. and Wellstead, A. (2016), "To bridge the divide between evidence and policy: reduce ambiguity as much as uncertainty”, Public Administration Review, Vol. 76 No. 3, pp. 399-402, doi: 10.1111/puar.12555.

Cambon, L., Petit, A., Ridde, V., Dagenais, C., Porcherie, M., Pommier, J., Ferron, C., Minary, L. and Alla, F. (2017), "Evaluation of a knowledge transfer scheme to improve policy making and practices in health promotion and disease prevention setting in French regions: a realist study protocol", Implementation Science, Vol. 12 No. 1, p. 83.

Canadian Foundation for Healthcare Improvement (2014), "Is research working for you? A selfassessment tool and discussion guide for health services management and policy organizations", Canadian Foundation for Healthcare Improvement, Ottawa, ON.

CIHR (2004), "Knowledge translation strategy 2004-2009: innovation in action", Canadian Institutes of Health Research, Ottawa, ON.

Darker, C.D., Nicolson, G.H., Carroll, A. and Barry, J.M. (2018), "The barriers and facilitators to the implementation of national clinical programmes in Ireland: using the MRC framework for process evaluations", BMC Health Services Research, Vol. 18 No. 1, p. 733, doi: 10.1186/s12913-018-3543-6.

Dew, A. and Boydell, K. (2017), "Knowledge translation: bridging the disability research-to-practice gap", Research and Practice in Intellectual and Developmental Disability, Vol. 4 No. 2, doi: 10.1080/ 23297018.2017.1315610.

Eden, C. and Ackermann, F. (1998), Making Strategy: The Journey of Strategic Management, Sage Publications, London.

Edwards, A., Zweigenthal, V. and Olivier, J. (2019), "Evidence map of knowledge translation strategies, outcomes, facilitators and barriers in African health systems", Health Research Policy and Systems, Vol. 17 No. 1, p. 16, doi: 10.1186/s12961-019-0419-0.

Elueze, I.N. (2015), "Evaluating the effectiveness of knowledge brokering in health research: a systematised review with some bibliometric information", Health Information \& Libraries Journal, Vol. 32 No. 3, pp. 168-181, doi: 10.1111/hir.12097. 
Fitzpatrick, A. (2012), "Working with parents in the early years services", in Mhic Mhathuna, M. and Taylor, M. (Eds), Early Childhood Education and Care. An Introduction for Students in Ireland, Gill and Macmillan.

Gagliardi, A.R., Berta, W., Kothari, A., Boyko, J. and Urquhart, R. (2016), "Integrated knowledge translation (IKT) in health care: a scoping review", Implementation Science, Vol. 11 No. 1, p. 38.

Geddes, M., Domnett, K. and Prosser, B. (2018), "A recipe for impact? Exploring knowledge requirements in the UK parliament and beyond", Evidence \& Policy: A Journal of Research, Debate and Practice, Vol. 14 No. 2, pp. 259-276, doi: 10.1332/174426417X14945838375115.

Green, T. (2019), "Publication is not enough, to generate impact you need to campaign", available at: https://blogs.Ise.ac.uk/impactofsocialsciences/2019/09/27/publication-is-not-enough-to-generateimpact-you-need-to-campaign/ (accessed 23 September 2019).

Grimshaw, J., Eccles, M., Lavis, J., Hill, S. and Squires, J. (2012), "Knowledge translation of research findings", Implementation Science: Is, Vol. 7, p. 50.

Growing Up in Ireland (2017), available at: www.twitter.com/GrowingUplre?lang=en (accessed 10 November 2017).

Grzymkowski, E. (2019), "2019 Google analytics benchmarks for higher education websites", available at: www.oho.com/blog/2019-google-analytics-benchmarks-higher-education-websites (accessed 15 September 2019)

Hickey, G., McGilloway, S., Leckey, Y. and Stokes, A. (2018), "A universal early parenting education intervention in community-based primary care settings: development and installation challenges", Education Sciences, Vol. 8 No. 4, p. 178.

Hickey, G., McGilloway, S., Leckey, Y., Stokes, A., O'Connor, S., Donnelly, M. and Bywater, T. (2020), "Exploring the potential utility and impact of a universal, multi-component early parenting intervention through a community-based, controlled trial", Children and Youth Services Review, Vol. 118, p. 105458, ISSN 0190-7409.

Hill, S. and McAlpine, H. (2019), "Knowledge exchange or research impact - what is the difference between REF and KEF?", available at: https://blogs.lse.ac.uk/impactofsocialsciences/2019/05/20/knowledgeexchange-or-research-impact-what-is-the-difference-between-ref-and-kef/ (accessed 15 September 2019).

Home, A., Carter, I., Scarth, S. and Warren, R. (2015), "Working together in special needs parenting: an innovative research dissemination project", Social Work \& Social Sciences Review, Vol. 17 No. 3, pp. 6-19, doi: 10.1921/12603170302.

Hutchings, J., Griffith, N., Bywater, T. and Williams, M.E. (2017), "Evaluating the incredible years toddler parenting programme with parents of toddlers in disadvantaged (flying start) areas of Wales", Child: Care, Health and Development, Vol. 43 No. 1, pp. 104-113.

Kernohan, W.G., Brown, M.J., Payne, C. and Guerin, S. (2018), "Barriers and facilitators to knowledge transfer and exchange in palliative care research", BMJ Evidence-Based Medicine, Vol. 23 No. 4, pp. 131-136, doi: 10.1136/bmjebm-2017-110865.

Kings College London and Digital Science (2015), "The Nature, Scale and Beneficiaries of Research Impact: An Initial Analysis of Research Excellence Framework (REF) 2014 Impact Case Studies", Higher Education Funding Council for England, London.

Landry, R., Amara, N. and Lamari, M. (2001), "Utilisation of social science research knowledge in Canada", Research Policy, Vol. 30 No. 2, pp. 333-349.

Leckey, Y., Hickey, G., Stokes, A. and McGilloway, S. (2019), "Parent and facilitator experiences of an intensive parent and infant programme delivered in routine community settings", Prim Health Care Res Dev, Vol. 20, p. e74, doi: 10.1017/S146342361900029X.

Leijten, P., Raaijmakers, M.A.J., Orobio de Castro, B., van den Ban, E. and Matthys, W. (2015), "Effectiveness of the incredible years parenting program for families with socioeconomically disadvantaged and ethnic minority backgrounds", Journal of Clinical Child \& Adolescent Psychology, Vol. 46 No. 1, pp. 59-73.

Li, S.A., Jeffs, L., Barwick, M. and Stevens, B. (2018), "Organizational contextual features that influence the implementation of evidence-based practices across healthcare settings: a systematic integrative review", Systematic Reviews, Vol. 7 No. 1, p. 72.

Lombardi, J. (2018), "What policymakers need from implementation evaluations of early childhood development programs", Annals of the New York Academy of Sciences, Vol. 1419 No. 1, pp. 17-19, doi: 10.1111/nyas. 13661. 
Mailchimp (2019), "Email marketing benchmarks and statistics by industry", available at: https:// mailchimp.com/resources/email-marketing-benchmarks (accessed 15 September 2019).

Margaryan, A., Littlejohn, A. and Vojt, G. (2011), "Are digital natives a myth or reality? University students' use of digital technologies", Computers \& Education, Vol. 56 No. 2, pp. 429-440, doi: 10.1016/j. compedu.2010.09.004.

Mee, G. (2019), "What is a good engagement rate on Twitter?", available at: www.scrunch.com/blog/ what-is-a-good-engagement-rate-on-twitter (accessed 15 September 2019).

Milat, A.J., King, L., Bauman, A. and Redman, S. (2011), "Scaling up health promotion interventions: an emerging concept in implementation science", Health Promotion Journal of Australia, Vol. 22 No. 3, p. 238.

Neiger, B.L., Thackeray, R., van Wagenen, S.A., Hanson, C.L., West, J.H., Barnes, M.D. and Fagen, M.C. (2012), "Use of social media in health promotion: purposes, key performance indicators, and evaluation metrics", Health Promotion Practice, Vol. 13 No. 2, pp. 159-164, doi: 10.1177/1524839911433467.

Ngamo, S.T., Souffez, K., Lord, C. and Dagenais, C. (2016), "Do knowledge translation (KT) plans help to structure KT practices?", Health Research Policy and Systems, Vol. 14 No. 1, p. 46, doi: 10.1186/s12961016-0118-z.

Oliver, K., Innvar, S., Lorenc, T., Woodman, J. and Thomas, J. (2014), "A systematic review of barriers to and facilitators of the use of evidence by policymakers", BMC Health Services Research, Vol. 14 No. 1.

Park, J.S., Moore, J.E., Sayal, R., Holmes, B.J., Scarrow, G., Graham, I.D., Jeffs, L., Timmings, C., Rashid, S., Johnson, A.M. and Straus, S.E. (2018), "Evaluation of the 'foundations in knowledge translation' training initiative: preparing end users to practice KT", Implement Sci, Vol. 13 No. 1, p. 63.

Powell, B.J., Beidas, R.S., Lewis, C.C., Aarons, G.A., McMillen, J.C., Proctor, E.K. and Mandell, D.S. (2017), "Methods to improve the selection and tailoring of implementation strategies", The Journal of Behavioral Health Services \& Research, Vol. 44 No. 2, pp. 177-194, doi: 10.1007/s11414-015-9475-6.

Stevens, B.J., Yamada, J., Estabrooks, C.A., Stinson, J., Campbell, F., Scott, S.D. and Cummings, G. (2014), "Pain in hospitalized children: effect of a multidimensional knowledge translation strategy on pain process and clinical outcomes", Pain, Vol. 155, pp. 60-68, doi: 10.1016/j. pain.2013.09.007.

Tricco, A.C., Antony, J., Soobiah, C., Kastner, M., Cogo, E., MacDonald, H., D’Souza, J., Hui, W. and Straus, S.E. (2016), "Knowledge synthesis methods for generating or refining theory: a scoping review reveals that little guidance is available", Journal of Clinical Epidemiology, Vol. 73, pp. 36-42.

Van Eerd, D. and Saunders, R. (2013), "Integrated knowledge transfer and exchange: an organizational approach for stakeholder engagement and communications", Health Res Policy Syst, Vol. 11, p. 13, doi: 10.22230/src.2017v8n1a274.

Wathen, C.N. and MacMillan, H.L. (2018), "The role of integrated knowledge translation in intervention research", Prevention Science, Vol. 19 No. 3, pp. 319-327, doi: 10.1007/s11121-015-0564-9.

Yamada, J., Shorkey, A., Barwick, M., Widger, K. and Stevens, B.J. (2015), "The effectiveness of toolkits as knowledge translation strategies for integrating evidence into clinical care: a systematic review", BMJ Open, Vol. 5 No. 4, p. e006808, doi: 10.1136/bmjopen-2014-006808.

Zhang, Z. and Ahmed, W. (2019), "A comparison of information sharing behaviours across 379 health conditions on Twitter", International Journal of Public Health, Vol. 64 No. 3, pp. 431-440.

\section{Further reading}

Barwick, M.A., Schachter, H.M., Bennett, L.M., McGowan, J., Ly, M., Wilson, A., Bennett, K., Buchanan, D.H., Fergusson, D. and Manion, I. (2012), "Knowledge translation efforts in child and youth mental health: a systematic review", Journal of Evidence-Based Social Work, Vol. 9 No. 4, pp. 369-395, doi: 10.1080/15433714.2012.663667.

\section{Corresponding author}

Siobhan O'Connor can be contacted at: siobhan.oconnor.2015@mumail.ie

For instructions on how to order reprints of this article, please visit our website: www.emeraldgrouppublishing.com/licensing/reprints.htm Or contact us for further details: permissions@emeraldinsight.com 